\title{
Profilassi eparinica nella tromboembolia polmonare: responsabilità professionale e profili giuridici
}

\author{
Dario Vizzotto ', Ennio Grassini ${ }^{2}$ \\ Medicina Legale, Università di Torino \\ Avvocato, Centro studi di diritto sanitario (www.dirittosanitario.net)
}

\begin{abstract}
Early diagnosis of deep vein thrombosis (DVT) is often difficult: DVT can be asimnthomatic and pulmonary thrombo-embolism (PTE) often occur in a sudden and unexpected way. Pharmacological prophylaxis, aiming at reducing the state of blood hypercoagulability, is the elective therapeutic strategy for vein thomboembolism. Pulmonary embolism is not only one of the most common causes of unexpected death in hospitalized patients, but also one of the top diseases leading to medical malpractice lawsuits. This article describes a case where the lack of adequate pharmacological prophylaxis caused the death of a woman for pulmonary thromboembolism. After some clinical and pharmacological considerations about prophylactic measures for PTE, the Authors explore the question of legal liability of medical practitioners in this situation.
\end{abstract}

Keywords: low-molecular-weight heparin (LMWH), pulmonary thrombo-embolism, professional liability

Heparin prophylaxis in pulmonary thrombo-embolism: professional responsibility and legal aspects. Pratica Medica \& Aspetti Legali 2007; 1(1): 13-22

\section{INTRODUZIONE}

Per trombosi venosa si intende l'ostruzione di un segmento venoso ad opera di un trombo. In base alla localizzazione dell'ostruzione si distinguono la trombosi venosa superficiale, o tromboflebite, quando è interessato il sistema venoso superficiale, e la trombosi venosa profonda, se è interessato il circolo venoso profondo degli arti.

La patogenesi dello sviluppo delle trombosi venose può essere ricondotta a tre principali fattori (triade Virchow):

- alterazioni delle caratteristiche fisico-chimiche del sangue (ipercoagulabilità);

- alterazioni del flusso ematico (stasi);

- alterazioni della parete vasale.

La trombosi venosa profonda (TVP) e la sua più temibile conseguenza, la tromboembolia polmonare (TEP), fatale, hanno registrato nelle ultime tre decadi un aumento dell'incidenza. I decessi causati da TEP sono spesso di interesse giudiziario per la frequente pre-esistenza di un evento traumatico e la repentinità della morte. Gli eventuali profili di colpa medica correlati possono derivare dall'assenza di profilassi adeguata, così come da una durata insufficiente della stessa. Ne consegue l'importanza del riconoscimento delle categorie di persone a rischio, di una diagnosi precoce, di una profilassi e terapia condotte correttamente, di un monitoraggio adeguato nel tempo.

\section{DESCRIZIONE DEL CASO}

Nel marzo 1999 il Tribunale di Foggia condannava gli imputati, un medico di guardia medica e tre dirigenti medici dipendenti di una ASL di Foggia, per il reato di cui all'art 589 del Codice penale (omici- 
dio colposo) per aver provocato la morte della signora $\mathrm{V}$.

Li condannava inoltre al risarcimento dei danni in favore delle parti civili costituitesi.

La Corte di Appello di Bari, investita della questione, dichiarava, nel novembre 2001, di non doversi procedere nei confronti dei predetti imputati perché il reato loro addebitato era da considerarsi estinto per prescrizione, confermando invece le statuizioni civili.

Si contestava agli imputati di aver cagionato la morte della paziente per embolia polmonare massiva.

In particolare al Dott. R., medico di guardia che aveva visitato la paziente in prima istanza, veniva contestato di aver omesso di formulare la diagnosi idonea non avendo rilevato la sospetta embolia polmonare di cui la signora V. riportava tutti i sintomi, avendo così determinato un ritardo nel ricovero e nell'inizio delle cure; al Dott. T., cardiologo in servizio presso il Pronto Soccorso, veniva contestato di non aver interpretato correttamente il tracciato dell'elettrocardiogramma della paziente in cui erano presenti tracce evidenti dell'embolia in corso; al Dott. P. e al Dott. C., medici di turno presso il presidio ospedaliero ove si era recata la paziente, di non aver approntato le cure necessarie nonostante fosse chiaramente diagnosticabile la citata patologia, essendosi limitati a consigliare il trasferimento presso altro ospedale, tra l'altro meno attrezzato.

La Corte era giunta alla conclusione che se la paziente fosse stata sottoposta a cure idonee e tempestive avrebbe senz'altro avuto l'opportunità di salvarsi.

\section{GLI ASPETTI CLINICI}

\section{FATTORI DA CUI DIPENDE IL RISCHIO TROMBOEMBOLICO}

La scelta del più adeguato regime di profilassi va effettuata sulla base di un attento inquadramento clinico del singolo caso e del rapporto rischio/benefici. In Tabella I è riportata la definizione dell'entità del rischio tromboembolico nel paziente chirurgico. I fattori di rischio in questo caso dipendono sia dalle caratteristiche del paziente sia dalla patologia concomitante. I fattori relativi al paziente comprendono:

- età (l'età > 60 anni è considerata un rischio aggiuntivo);

- costituzione (obesità);

- uso di estroprogestinici (vanno sospesi almeno un mese prima);

- altre patologie presentate dal paziente;

- cardiopatie (protesi valvolari cardiache, fibrillazione atriale, difetti valvolari, cardiopatia ischemica, miocardiopatia dilatativa);

- pregressi episodi tromboembolici;

- alterazioni ematologiche con tendenza alla trombofilia (poliglobulia, trombocitosi, malattie mieloproliferative, deficit di proteina $\mathrm{C}$, S, LAC/APLA, AT III, ecc.);

- varici arti inferiori.

- BOC severa;

- patologia neoplastica maligna;

- patologie che impediscono la mobilità degli arti inferiori (es. ictus, lesioni del midollo spinale, politraumi).

\begin{tabular}{|c|c|c|c|}
\hline Rischio & Età & Fattori di rischio & Intervento \\
\hline Basso & Età $<40$ aa & Senza fattori di rischio* & Chirurgia minore non complicata \\
\hline \multirow[t]{3}{*}{ Moderato } & Età $<40$ aa & Senza fattori di rischio & Chirurgia maggiore/malignità \\
\hline & Età compresa tra 40 e 60 aa & Senza fattori di rischio & Chirurgia di ogni tipo \\
\hline & Qualunque** & Con fattori di rischio & Chirurgia minore \\
\hline \multirow[t]{2}{*}{ Elevato } & Età compresa tra 40 e 60 aa & Con fattori di rischio & Chirurgia maggiore/malignità \\
\hline & $>60 \mathrm{aa}$ & Senza fattori di rischio agg & Chirurgia maggiore/malignità \\
\hline \multirow[t]{4}{*}{ Elevatissimo } & Età $>40$ aa & Pregressa tromboembolia & Chirurgia maggiore/malignità \\
\hline & Età $>40$ aa & Stato ipercoagulativo & Chirurgia maggiore/malignità \\
\hline & Qualsiasi & & $\begin{array}{l}\text { Chirurgia maggiore ortopedica in } \\
\text { elezione dell'anca e del ginocchio }\end{array}$ \\
\hline & Qualsiasi & & $\begin{array}{l}\text { Chirurgia ortopedica d'urgenza } \\
\text { per frattura dell'anca/pelvi, } \\
\text { patellectomia }\end{array}$ \\
\hline
\end{tabular}

Tabella I. Definizione dell'entità del rischio tromboembolico nel paziente chirurgico

* Ia coesistenza di uno o più fattori aggiuntivi di rischio sposta i pazienti dalla categoria inferiore di rischio immediatamente a quella superiore

** I'età > 60 aa è considerata fattore di rischio per gli interventi in anestesia generale o spinale/epidurale 
I fattori di rischio relativi alla patologia che richiede l'intervento chirurgico e al tipo di intervento sono:

- patologia neoplastica maligna, stato settico;

- chirurgia maggiore (anche laparoscopica) e chirurgia complicata (ad esempio settica, emorragica);

- estese dissezioni (ad esempio alcuni interventi di chirurgia plastica, ampie dissezioni linfonodali);

- sede dell'intervento (ad esempio in tutta la chirurgia pelvica: urologica prostatica e vescicale, ginecologica, ortopedica);

- durata dell'intervento (> $30 \mathrm{~min}$ );

- interventi che presuppongono una immobilizzazione prolungata (oppure una deambulazione tardiva e/o insufficiente).

\section{PROFILASSI}

La diagnosi precoce della TEV, e quindi del rischio di TEP ad essa connesso, è spesso di difficile attuazione, poiché la TEV può essere completamente asintomatica e la TEP spesso insorge in modo improvviso e imprevedibile poco prima del decesso, rendendo impossibile intraprendere un intervento terapeutico efficace. Per questi motivi la profilassi rappresenta la strategia terapeutica elettiva per questo tipo di patologia (Tabella II e Tabella III).

La profilassi può essere condotta farmacologicamente o con mezzi fisici come la compressione elastica (graduata o pneumatica intermittente) o con l'interruzione parziale della vena cava inferiore (limitatamente a casi particolari).

Attualmente i farmaci utilizzati per la profilassi farmacologica comprendono le eparine, il dermatan solfato, gli anticoagulanti orali e gli antiaggreganti piastrinici. Non esiste una linea guida unica per tutte le specializzazioni chirurgiche, così come in molti Centri le linee guida di una determinata branca chirurgica (urologia, ginecologia, ecc.) possono essere per certi versi dissimili da quelle di analoghi reparti.

La scelta del tipo di profilassi dipende quindi essenzialmente dall'entità del rischio e dalle possibili controindicazioni alle singole metodiche.

\section{Linee guida di profilassi tromboembolica in chirurgia}

\section{Profilassi con mezzi fisici}

Bendaggio fisso degli arti inferiori e calze elastiche antitrombo:

- metodiche controindicate in caso di arteriopatia ostruttiva degli arti inferiori;

- presidi particolarmente indicati in caso di sindrome post-flebitica, insufficienza venosa cronica di rilievo;

- opportuno sottolineare l'importanza della giusta scelta della taglia della calza (piccola, media, grande; corta o lunga);

Compressione pneumatica intermittente degli arti inferiori:

- secondo la letteratura sembra avere una efficacia sovrapponibile se non superiore alla terapia farmacologica;

- indispensabile nei casi in cui sia controindicato l'impiego dell'eparina, in associazione o meno alle calze elastiche antitrombo (ad esempio pazienti sottoposti ad intervento neurochirurgico intracranico).

L'impiego di un filtro cavale temporaneo deve essere preso in considerazione qualora si dovesse procedere ad un intervento chirurgico:

- in urgenza, in un paziente con flebotrombosi in atto;

- in paziente con altissimo rischio per evento tromboembolico;

- in urgenza ortopedica, in un paziente ad alto rischio tromboembolico e con sanguinamento in atto, che controindica l'impiego di farmaci anticoagulanti (ad esempio nel caso di frattura del bacino).

Una precoce mobilizzazione del paziente nel postoperatorio, con una conseguente soddisfacente deambulazione quando possibile, rappresentano una delle misure più importanti per la prevenzione delle tromboembolie. Una adeguata terapia antidolorifica nell'immediato post-operatorio è di aiuto a tal fine.

\section{Profilassi farmacologica eparinica}

Molteplici studi randomizzati hanno dimostrato che le eparine costituiscono la classe farmacolo-

\begin{tabular}{|lc|}
\multicolumn{1}{|c|}{ Condizione } & Incidenza \\
\hline $\begin{array}{l}\text { In chirurgia generale (chirurgia laparotomica } \\
\text { maggiore o mastectomia) } \\
\text { In paziente di età > } 40 \text { anni e } \\
\text { durata dell'intervento > } 30 \text { minuti }\end{array}$ & $25 \%$ \\
\hline Nella chirurgia protesica dell'anca & \\
\hline In chirurgia ortopedica per frattura del femore & $51 \%$ \\
\hline Nell'artroprotesi del ginocchio & $48 \%$ \\
\hline
\end{tabular}

Tabella II. Incidenza della trombosi venosa profonda (TVP), senza profilassi

Grado di rischio

\begin{tabular}{|lc|}
\hline Rischio basso & $0,2 \% / 0,002 \%$ \\
\hline Rischio moderato & $1-2 \% / 0,1-0,4 \%$ \\
\hline Rischio elevato & $2-4 \% / 0,4-1 \%$ \\
\hline Rischio elevatissimo & $4-10 \% / 1-5 \%$ \\
\hline
\end{tabular}

Tabella III. Incidenza dell'embolia polmonare (EP) clinicamente manifesta ed embolia polmonare fatale rispetto al grado di rischio tromboembolico in chirurgia e condizioni mediche 
gica di maggiore efficacia. Esistono in commercio diversi preparati eparinici:

- eparina non frazionata a basse dosi (fisse) per via sottocutanea (LDUH, Low Dose Heparin, $5.000 \mathrm{UI} / 12$ ore; $5.000 \mathrm{UI} / 8$ ore), rivelatasi efficace in pazienti chirurgici e internistici;

- eparina standard in dosi "aggiustate" in base ai valori del tempo di tromboblastina parziale. Nonostante l'efficacia lo schema profilattico usato in terapia ortopedica non ha trovato una diffusa applicazione pratica;

- eparine a basso peso molecolare (LMWH, Low Molecular Weight Heparin, 4.000-6.500 Da), ottenute attraverso la degradazione chimica o enzimatica dell'eparina.

In generale le LMWH costituiscono i farmaci di elezione grazie alla più lunga emivita plasmatica, alla maggiore biodisponibilità, ai minori effetti collaterali e della praticità del trattamento (somministrazione in dosi fisse, senza necessità di controlli dei parametri emocoagulativi). Le formulazioni di LMWH presenti in Italia sono: bemiparina, calciparina, dalteparina, enoxaparina, nadroparina, parnaparina e reviparina.

Le eparine a basso peso molecolare differiscono per il metodo impiegato nella produzione, nel peso molecolare e nella attività specifica. Si raccomanda pertanto di attenersi alle rispettive schede tecniche e di non passare da un marchio all'altro durante il trattamento.

Generalmente la dose giornaliera di LMWH, eseguita per la profilassi tromboembolica, va praticata in un'unica somministrazione.

Qualora il dosaggio giornaliero dovesse essere superiore alle 4.000 UI di enoxaparina (o dosaggi equivalenti delle altre eparine a basso peso molecolare) oppure quando sia auspicabile un'azione costante del farmaco nel tempo (come in caso di cardiopatia ischemica o congestizia, nei portatori di protesi valvolari cardiache, nei pazienti con fibrillazione atriale, nei pazienti ad elevatissimo rischio tromboembolico), può essere consigliabile frazionare la dose giornaliera in due somministrazioni/die.

Un altro aspetto molto importante in chirurgia generale riguarda l'epoca ottimale di somministrazione sia di LDUH che di LMWH. Negli interventi di chirurgia generale, eccetto quelli eseguiti in anestesia spinale o peridurale, con previsione di un normale rischio di sanguinamento intraoperatorio, la somministrazione di LMWH ad un dosaggio non superiore alle $2.000 \mathrm{UI}$ (enoxaparina) può essere effettuata fino a due ore prima dell'inizio dell'intervento chirurgico, in modo tale da contrastare l'attivazione della cascata emocoagulativa che ha luogo nel corso dell'intervento e prevenire in tal modo la formazione iniziale del trombo. In tutti gli interventi di chirurgia ortopedica e negli interventi di chirurgia generale con previsione di un rischio emorragico intraoperatorio elevato o molto elevato, la somministrazione di LMWH può essere iniziata 12 ore prima, e comunque non meno di 8 ore prima, dell'intervento chirurgico, oppure direttamente nel post-operatorio dalle 6 alle 12 ore dopo l'intervento stesso, eventualmente associata ad altre metodiche di profilassi.

Lassociazione dell'eparina a basso peso molecolare è sconsigliata nel caso di assunzione concomitante di altri farmaci quali:

- acido acetilsalicilico o altri salicilati;

- FANS (farmaci anti-infiammatori non steroidei);

- ticlopidina;

- altri antiaggreganti piastrinici (dipiridamolo, sulfinpirazone).

Questi farmaci vanno sospesi alcuni giorni prima dell'intervento (dai 3 ai 6 giorni secondo il tipo di farmaco) e sostituiti con l'eparina a basso peso molecolare, in mono o bi-somministrazione giornaliera, secondo i casi.

Anche i pazienti in terapia con altri anticoagulanti (antagonisti della vitamina $\mathrm{K}$, ad esempio acenocumarolo e warfarin) devono sospendere l'assunzione di questi farmaci almeno tre giorni prima dell'intervento sostituendoli con l'eparina a basso peso molecolare fino a valori nell'INR, eseguito al mattino prima della somministrazione dell'eparina, compatibili con l'intervento.

Nel post-operatorio alla ripresa della terapia con acenocumarolo o warfarin, è consigliato sovrapporre la terapia con l'eparina controllando sempre giornalmente i valori della coagulazione (INR o PT e PTT) fino al raggiungimento del valore terapeutico dell'INR (o del PT) per il paziente; successivamente sospendere l'eparina e continuare con la sola terapia anticoagulante per os. Si consiglia di riprendere la somministrazione dei dicumarolici con un dosaggio uguale a quello assunto dal paziente prima dell'intervento.

Si consiglia inoltre di somministrare i dicumarolici alle ore 18.00 per essere a conoscenza del valore dell'INR del giorno stesso (in genere eseguito alle ore 6.00) e per avere un attendibile valore della coagulazione della mattina successiva.

Nelle normali condizioni d'impiego per la profilassi tromboembolica l'eparina a basso peso molecolare non modifica i parametri della coagulazione. Qualora fosse utilizzata per un trattamento prolungato (ad esempio protratta immobilità del paziente per complicanze locali o generali) e a dosaggi elevati, sarà opportuno eseguire un monitoraggio biologico che consiste nel controllo frequente della conta piastrinica. Ovviamente una conta piastrinica sarà stata eseguita prima del trattamento. Sono stati descritti, infatti, rari casi di trombocitopenia iatrogena, talvolta gravi.

L' uso delle eparine è controindicato in caso di:

- gravi alterazioni dei parametri della coagulazione;

- manifestazioni o tendenze emorragiche legate a disturbi dell'emostasi;

- gravi epatopatie;

- lesioni organiche a rischio di sanguinamento (ad esempio ulcera gastroduodenale in fase 
attiva, traumi cranioencefalici e accidenti cerebrovascolari emorragici);

- endocardite infettiva acuta;

- anamnesi positiva per trombocitopenia iatrogena;

- allergia alle eparine.

Particolare attenzione andrà attuata in caso di anestesia spinale/epidurale. L'inserimento dell'ago a livello spinale dovrebbe essere effettuato almeno 10-12 ore dopo la somministrazione dell'eparina a basso peso molecolare, così come il cateterino epidurale andrebbe rimosso non prima di 10 ore dalla precedente somministrazione di eparina e almeno due ore prima dalla successiva somministrazione del farmaco (per esempio nel caso di una somministrazione alle ore 6.00 e alle ore 18.00 , il cateterino può essere rimosso alle ore 16.00).

Le modalità di somministrazione prevedono:

- via sottocutanea con ago sottile (siringhe già pronte per l'eparina a basso peso molecolare);

- alternare i punti di inoculo ad ogni somministrazione;

- in caso di chirurgia addominale, nell'immediato pre-operatorio inoculazione all'esterno della spina iliaca per non interessare il campo operatorio;

- nel post-operatorio iniezioni lontano dai drenaggi e dalla ferita chirurgica.

La somministrazione di eparina a basso peso molecolare andrà effettuata fino alla mobilizzazione completa del paziente (in genere 7-10 giorni per il paziente di chirurgia generale e fino a tre settimane per il paziente ortopedico), cioè fino ad una soddisfacente deambulazione.

Nel caso tale periodo di rischio tromboembolico si dovesse prolungare (o nel caso di artroprotesi totale di ginocchio, o per l'insorgenza di complicanze locali o generali, o per pre-esistenti cause mediche di rischio tromboembolico) sarà necessario un trattamento di più lunga durata oppure il ricorso ad altre metodiche di profilassi tromboembolica (compressione pneumatica intermittenete degli arti inferiori o anticoagulanti orali, secondo il caso clinico).

\section{Profilassi antitrombotica consigliata nel paziente di chirurgia generale, urologica e plastica}

\section{Paziente a basso e medio rischio:}

- eparina a basso peso molecolare, a un dosaggio minore ${ }^{1}$, da praticare preferibilmente 12 ore prima dell'intervento oppure

\footnotetext{
enoxaparina 2.000 UI o dosaggi equivalenti delle altre eparine a basso peso molecolare: bemiparina $2.500 \mathrm{UI}$, calciparina $5.000 \mathrm{UI}$, dalteparina $2.500 \mathrm{UI}$, nadroparina 2.850 UI, parnaparina $3.200 \mathrm{UI}$, reviparina $1.750 \mathrm{UI}$
}

- eparina calcica a basse dosi: calciparina 5.000 UI $x 2$ sc

oppure

- in alcuni casi, calze antitrombo (ad esempio paziente a basso rischio, di età $<40$ anni, sottoposto ad intervento di chirurgia viscerale, non neoplastica, non infiammatoria e senza estese dissezioni);

- la sola mobilizzazione precoce all'inizio della stessa giornata dell'intervento può essere ritenuta sufficiente, se effettivamente possibile per la sede e il tipo di intervento chirurgico, solo nel paziente a basso rischio (pertanto senza rischi aggiuntivi tromboembolici) sottoposto ad intervento di durata $<30$ minuti (generalmente interventi ambulatoriali o in regime di Day Surgery in anestesia locale).

\section{Paziente a rischio elevato:}

- eparina a basso peso molecolare (LMWH) ad un dosaggio minore (es: enoxaparina 2.000 $\mathrm{UI} /$ die o dosaggi equivalenti delle altre eparine a basso peso molecolare) + calza antitrombo o bendaggio fisso degli arti inferiori o CPI

oppure

- eparina a basso peso molecolare ad un dosaggio maggiore ${ }^{2}$ (es: enoxaparina $4.000 \mathrm{UI} /$ die).

\section{Paziente a rischio elevatissimo:}

- eparina a basso peso molecolare ad un dosaggio maggiore (es. enoxaparina $4.000 \mathrm{UI} /$ die) + calza antitrombo o bendaggio fisso arti inferiori o compressione pneumatica intermittente degli arti inferiori

oppure

- compressione pneumatica intermittente degli arti inferiori, eventualmente associata alle calze antitrombo, nei casi in cui sia controindicato l'uso delle eparine.

Nei pazienti ad altissimo rischio tromboembolico (ad esempio con pregressi episodi tromboembolici o stati trombofilici) le modalità di profilassi vanno attuate secondo indicazioni ben precise, valutate caso per caso, concordate dal chirurgo con lo specialista angiologo e/o cardiologo e/o radiologo vascolare. Comunque non vanno mai somministrate dosi inferiori a 4.000 UI di enoxaparina o equivalenti, talvolta anche superiori se non controindicata, associate a calze antitrombo o bendaggio fisso degli arti inferiori o compressione pneumatica intermittente.

\footnotetext{
2 enoxaparina 4.000 UI o dosaggi equivalenti delle altre eparine a basso peso molecolare: bemiparina $3.500 \mathrm{UI}$, calciparina $12.500 \mathrm{UI}$, dalteparina 5.000 UI, nadroparina 40-60 $\mathrm{UI} / \mathrm{kg}$ (il dosaggio deve essere modificato secondo la giornata post-operatoria, vedi scheda tecnica), parnaparina 4.250 UI, reviparina $4.200 \mathrm{UI}$
} 
Il filtro cavale è riservato ad alcuni casi ben selezionati di pazienti a rischio elevatissimo (con flebotrombosi in atto, chirurgia ortopedica maggiore dell'anca emorragica in paziente ad altro rischio tromboembolico).

La profilassi con eparina andrà protratta per almeno una settimana o fino ad una accettabile deambulazione.

\section{Profilassi antitrombotica consigliata nel paziente di chirurgia ortopedica}

Paziente a basso rischio (interventi minori del ginocchio, es artroscopia, e degli arti superiori in assenza di rischi aggiuntivi):

- eparina a basso peso molecolare, ad un dosaggio minore ${ }^{3}$, da praticare preferibilmente 12 ore prima dell'intervento

oppure

- eparina calcica a basse dosi: calciparina 5.000 UI $x 2$ sc

oppure

- la sola mobilizzazione precoce all'inizio dalla stessa giornata dell'intervento può essere ritenuta sufficiente, se effettivamente possibile per la sede e il tipo di intervento chirurgico, solo nel paziente a basso rischio (pertanto senza rischi aggiuntivi tromboembolici) sottoposto a intervento di durata $<30 \mathrm{mi}$ nuti (generalmente interventi degli arti superiori in regime ambulatoriale o in regime di Day Surgery in anestesia locale).

\section{Paziente a rischio moderato:}

- eparina a basso peso molecolare, ad un dosaggio minore ${ }^{3}$, da praticare preferibilmente 12 ore prima dell'intervento + calza antitrombo o bendaggio fisso degli arti inferiori o CPI.

\section{Paziente a rischio elevato:}

- eparina a basso peso molecolare ad un dosaggio maggiore ${ }^{4}$ (es: enoxaparina $4.000 \mathrm{UI} /$ die o dosaggi ancora superiori)

oppure

- compressione pneumatica intermittente degli arti inferiori, eventualmente associata alle calze antitrombo, nei casi in cui sia controindicato l'uso delle eparine.

\footnotetext{
${ }^{3}$ enoxaparina 2.000 o dosaggi equivalenti delle altre eparine a basso peso molecolare: bemiparina $2.500 \mathrm{UI}$, calciparina $5.000 \mathrm{UI}$, dalteparina $2.500 \mathrm{UI}$, nadroparina $2.850 \mathrm{UI}$, parnaparina $3.200 \mathrm{UI}$, reviparina $1.750 \mathrm{UI}$

${ }^{4}$ enoxaparina 4.000 UI o dosaggi equivalenti delle altre eparine a basso peso molecolare: bemiparina $3.500 \mathrm{UI}$, calciparina $12.500 \mathrm{UI}$, dalteparina 5.000 UI, nadroparina 40-60 $\mathrm{UI} / \mathrm{kg}$ (il dosaggio deve essere modificato secondo la giornata post-operatoria, vedi scheda tecnica), parnaparina 4.250 UI, reviparina $4.200 \mathrm{UI}$
}

Pazienti ad elevatissimo rischio (flebotrombosi in atto, flebotrombosi recidivanti, pregressa embolia polmonare recidivante, stati trombofilici):

- eparina a basso peso molecolare ad un dosaggio maggiore (es: enoxaparina $4.000 \mathrm{UI} /$ die o dosaggi ancora superiori) + calza antitrombo o bendaggio fisso degli arti inferiori oppure compressione pneumatica intermittente;

- compressione pneumatica intermittente degli arti inferiori, associata alle calze antitrombo, nei casi in cui sia controindicato l'uso delle eparine.

Nei pazienti ad altissimo rischio tromboembolico (pregressi episodi tromboembolici, stati trombofilici) le modalità di profilassi vanno attuate secondo indicazioni ben precise, valutate caso per caso, concordate dal chirurgo con lo specialista angiologo e/o cardiologo e/o radiologo vascolare. Comunque non meno di 4.000 UI di enoxaparina (o equivalenti), talvolta dosaggi ancora superiori, se non controindicati, associati a calze antitrombo o bendaggio fisso degli arti inferiori o compressione pneumatica intermittente.

Il filtro cavale è riservato ad alcuni casi ben selezionati di pazienti a rischio elevatissimo (con flebotrombosi in atto o in caso di chirurgia ortopedica maggiore dell'anca emorragica in pazienti ad altro rischio tromboembolico).

La profilassi con eparina andrà protratta per almeno tre settimane $o$, comunque, fino a una accettabile deambulazione.

\section{CONCLUSIONI}

In conclusione, la profilassi della TVP, in previsione di un intervento chirurgico, può essere condotta con eparina non frazionata a basse dosi o con eparina frazionata a basso peso molecolare.

L'eparina non frazionata a basse dosi (5.000 UI x 2-3/die sc) si dimostra efficace nel diminuire l'incidenza di TVP e di EP fatale.

Le eparine LMWH si dimostrano efficaci quanto o più l'eparina non frazionata nella prevenzione di TVP ed EP fatale in chirurgia generale e ortopedica, utilizzate a dosi proporzionate al peso corporeo e senza necessità di monitoraggio nei parametri coagulativi.

La durata delle profilassi, per tutte le categorie di pazienti con diverso grado di rischio, si protrae finché persiste l'immobilizzazione e comunque per non meno di 7-10 giorni.

Attualmente le raccomandazioni per la profilassi operatoria della TVP sono riassunte nelle varie linee guida; va tuttavia ricordato che nuovi studi comparativi si aggiungono rapidamente nel campo della prevenzione, e ogni suggerimento ha un valore temporaneo e non sembra destinato a durare a lungo. 
Attualmente le LMWH sono efficaci e sicure nella profilassi del tromboembolismo venoso in chirurgia generale e presentano evidenti vantaggi nei confronti dell'eparina non frazionata a basse dosi. Tale metodica è indubbiamente potenziata dall'uso contemporaneo di calze elastiche antitrombo.

Nei casi in cui esista una controindicazione alla somministrazione di eparina per condizioni cliniche generali, in previsione di un intervento chirurgico verrà presa in considerazione la possibilità di interruzione parziale della vena cava inferiore (VCI) con un filtro temporaneo o definitivo a seconda che il rischio emboligeno sia limitato o permanga nel tempo.

\section{GLI ASPETTI LEGALI}

\section{COMPORTAMENTO CENSURATO}

La causa della morte della paziente era da identificarsi nella insorgenza di una tromboembolia sub-massiva non riconosciuta dai curanti; la paziente conseguentemente veniva sottoposta al trattamento terapeutico (eparina e/o trombolitici) quando ormai era inutile ogni tipo di intervento. Dal decorso tipico, lento, manifesto, progressivamente ingravescente della malattia la Corte deduceva che una tempestiva instaurazione di appropriata terapia, pure in assenza di conferme diagnostiche, avrebbe salvato la paziente o, quanto meno, le avrebbe dato le opportunità di salvezza che di fatto sono state precluse.

La stessa Corte D’Appello rilevava: "pacifico deve ritenersi che la terapia elettiva della TEP (tromboembolia polmonare), idonea a bloccare l'evoluzione della malattia e, se tempestiva, a salvare la vita al paziente, è la somministrazione di eparina per via endovenosa, terapia che, secondo l'univoco insegnamento dei più autorevoli studiosi della malattia, incontestabilmente condiviso nel campo scientifico ed operativo, dovrebbe essere istituita immediatamente, in presenza di un elevato sospetto di embolia, senza attendere una conferma diagnostica, a meno che la dose iniziale di eparina non esponga il paziente a rischi evidenti».

\section{ATTEGGIAMENTO CONSIGLIATO}

La Corte di Cassazione nel caso esaminato in premessa, secondo quanto emerso dalle perizie effettuate, aveva ritenuto che la sintomatologia della paziente avrebbe senz'altro "dovuto orientare il professionista verso la corretta diagnosi al fine di non incorrere nel grave e non scusabile errore diagnostico ascrivibile a superficialità ed imperizia» [1]. Deve però tenersi conto del fatto che spesso la diagnosi non è semplice da formulare. Ad esem- pio nel caso trattato, i sintomi della embolia polmonare non erano univoci, bensì subdoli e transitori (secondo quanto riferito dagli stessi medici imputati) «per cui risultava impossibile anche per medici di spiccata abilità e perizia tecnica formulare la diagnosi senza l'ausilio di idonea strumentazione».

Come può dedursi dalla generalità dei casi di responsabilità trattati dalla Suprema Corte, i medici sono spesso accusati di omessa diligente e tempestiva diagnosi, con conseguente ritardo nelle cure, che se "doverosamente realizzate sarebbero state idonee ad evitare la progressiva involuzione letale della paziente con alto grado di probabilità logica», nonché talvolta di lacunoso monitoraggio laboristico e strumentale [2]. Tuttavia in questo tipo di giudizio, la prova della sussistenza del nesso di causalità tra azione o omissione ed evento lesivo, deve essere rigorosa; ai fini del profilo di colpevolezza, risulta necessario provare in maniera scientifica «l'esatto limite temporale entro cui se la paziente fosse stata sottoposta ad un trattamento con eparina e/o trombolitici si sarebbe salvata».

\section{OSSERVAZIONI}

Appare opportuno segnalare il contrasto giurisprudenziale sorto in relazione al nesso di causalità tra azione od omissione e l'evento morte necessario affinché l'imputato possa essere ritenuto colpevole del reato ascrittogli.

Infatti nel caso de quo, la Corte D’Appello di Bari riteneva che «al criterio della certezza si deve sostituire quello della probabilità per cui il rapporto causale sussiste anche quando l'opera del medico, se correttamente e tempestivamente intervenuta, avrebbe avuto serie ed apprezzabili possibilità di successo, tali che la vita della paziente sarebbe stata salvata» [3].

Al contrario la Corte di Cassazione [4] investita della medesima questione, sosteneva, come già ritenuto dalle Sezioni Unite di questa Suprema Corte (sentenza n. 30328/2002, Franzese), che hanno in tal guisa risolto il contrasto giurisprudenziale sul punto, che non è consentito dedurre automaticamente dal coefficiente di probabilità espresso dalla regola statistica la conferma, o meno, del-

Negligenza: omesso compimento di un'azione doverosa Nel trattamento della TEP $\rightarrow$ omissione di inquadramento clinico relativo a eventuali fattori di rischio per TVP

Imprudenza: inosservanza di un divieto assoluto di agire o di un divieto di agire secondo determinate modalità Nel trattamento della TEP $\rightarrow$ sospensione di un trattamento profilattico per la TEP in paziente a rischio

Imperizia: negligenza 0 imprudenza in attività che richiedono l'impiego di particolari abilità o cognizioni Nel trattamento della TEP $\rightarrow$ omissione del trattamento profilassico eparinico in presenza di chiare indicazioni 
l'ipotesi accusatoria sull'esistenza del nesso causale, poiché il giudice deve verificare la validità nel caso concreto, sulla base delle circostanze del fatto e dell'evidenza disponibile, così che, all'esito del ragionamento probatorio, che abbia altresì escluso l'interferenza di fattori alternativi, risulti giustificata e processualmente certa la conclusione che la condotta omissiva del medico è stata condizione necessaria dell'evento lesivo con "alto o elevato grado di credibilità razionale o probabilità logica». In sostanza, con la tralaticia formula delle «serie ed apprezzabili probabilità di successo" dell'ipotetico intervento salvifico del medico, «si finisce per esprimere coefficienti di probabilità indeterminati, mutevoli, manipolabili dall'interprete, talora attestati su standard davvero esigui», mentre, "poiché la condizione necessarietà si configura come requisito oggettivo della fattispecie criminosa, non possono non valere per essa gli stessi identici criteri di accertamento e di rigore dimostrativo, che il giudizio penale riserva a tutti gli elementi costitutivi del fatto di reato", dovendo il procedimento logico "condurre" alla conclusione caratterizzata da un alto grado di credibilità razionale, quindi alla "certezza processuale".

\section{BIBLIOGRAFIA}

\section{Parte clinica}

1. Agnelli G, Piovella F, Buoncristiani P, et al. Enoxaparin plus compression stockings compared with compression stockings alone in the prevention of venous thromboembolism after elective neurosurgery. $N$ Engl J Med 1998; 339: 80-5

2. Arcasoy SM, Kreit JW. Thrombolytic therapy of pulmonary embolism. A comprehensive review of current evidence. Chest 1999; 115: 1695-707

3. Casazza F, Bongarzoni A, Centonze F et al. Prevalence and prognostic significance of right-sided cardiac mobile thrombi in acute massive pulmonary embolism. Am J Cardiol 1997; 79: 1433-5

4. Clarke-Pearson DL, Coleman RE, Synan IS et al. VTE prophylaxis in gynecologic oncology: a prospective controlled trial of lowdose heparin. Am J Obstet Gynecol 1983; 145: 606-13

5. Clarke-Pearson DL, De Long E, Synan IS et al. A controlled trial of two low-dose heparin regimens for the prevention of postoperative deep vein thrombosis. Obstet Gynecol 1990; 75: 684-9

6. Clarke-Pearson DL, Synan IS, Dodge R et al. A randomized trial of low-dose heparin and intermittent pneumatic calf compression for the prevention of deep vein thrombosis after gynecologic oncology surgery. Am J Obstet Gynecol 1993; 168: 1146-54

7. Cohen SH, Erhlich GE, Kauffman MS et al. Thrombophlebitis following knee surgery. J Bone Joint Surg Am 1973; 55: 106-12

8. Columbus Investigators. Low-molecular-weight heparin in the treatment of patients with venous thromboembolism. $N$ Engl $J$ Med 1997; 337: 657-62

9. Cuccia C, Campana M, Franzoni P et al. Effectiveness of intravenous rtPA in the treatment of massive pulmonary embolism and right heart thromboembolism. Am Heart J 1993; 126: 468-72

10. Etchells E, McLeod RS, Geerts W, Barton P, Detsky AS. Economic analysis of low-dose heparin vs low-molecular weight heparin enoxaparin for prevention of venous thromboembolism after colorectal surgery. Arch Intern Med 1999; 159: 1221-8

11. Zonzin P, Agnelli G, Casazza F, Favretto G, Giuntini C, Morpurgo M, Vizza CD. Comments on the guidelines of the European Society of Cardiology Task Force on pulmonary embolism. Ital Heart J 2001; 2: 1342-56

12. Garlund B, for the Heparin Prophylaxis Study Group. Randomised controlled trial of low-dose heparin for prevention of fatal pulmonary embolism in patients with infectious diseases. Lancet 1996; 347: 1357-61

13. Geerts WH, Heit JA, Clagett GP et al. Prevention of venous thromboembolism. Chest 2001; 119: 132S-175S

14. Ginsberg JS, Hirsh J. Use of antithrombotic agents during pregnancy. Chest 1992; 102: 385S-390S

15. Giuntini C, Di Ricco G, Marini C et al. Pulmonary embolism: epidemiology. Chest 1995; 107: 3S-9S

16. Goldhaber SZ, Heit J, Sharma GV et al. Randomised controlled trial of recombinant tissue plasminogen activator versus urokinase in the treatment of acute pulmonary embolism. Lancet 1988; 2: 293-8

17. Grifoni S, Olivotto I, Cecchini P et al. Short-term clinical outcome of patients with acute pulmonary embolism, normal blood pressure, and echocardiographic right ventricular dysfunction. Circulation 2000; 101: 2817-22

18. AA.VV. Guidelines 2000 for cardiopulmonary resuscitation and emergency cardiovascular care: international consensus on science. Circulation 2000; 102: I1-I384

19. Haake DA, Berkman SA. Venous thromboembolic disease after hip surgery: risk factors, prophylaxis and diagnosis. Clin Orthop 1989; 242: 212-31

20. Heinzer H, Hammerer P, Graefen M, Huland $\mathrm{H}$. Thromboembolic complication rate after radical retropubic prostatectomy: impact of routine ultrasonography for the detection of pelvic lymphoceles and hematomas. Eur Urol 1998; 33: 86-90

21. Heit JA, Elliott CG, Trowbridge AA et al. Ardeparin sodium for extended out-of-hospital prophylaxis after total hip or knee replacement: a randomised, double blind, placebo controlled trial. Ann Intern Med 2000; 132: 853-61

22. Heit JA, Silverstein MD, Mohr DN et al. Predictors of survival after deep vein thrombosis and pulmonary embolism: a population-based, cohort study. Arch Intern Med 1999; 159: 445-53 
23. Hills NH, Pflug JJ, Jeyasingh $\mathrm{K}$ et al. Prevention of deep vein thrombosis by intermittent pneumatic compression of calf. $B M J$ 1972; 1: 131-5

24. Hirsh T, Warkentin TE, Shanghnessy SG et al. Heparin and low-molecular weight heparin. Chest 2001; 119: 64S-94S

25. Hoek JA, Nurmohamed MT, Hamelynck KJ et al. Prevention of deep vein thrombosis following total hip replacement by low-molecular weight heparinoid. Thromb Haemost 1992; 67: 28-32

26. Horlocker TT, Heit JA. Low molecular weight heparin: biochemistry, pharmacology, perioperative prophylaxis regimens and guidelines for regional anesthetic management. Anesth Analg 1997; 85: 874-85

27. Hyers TM, Agnelli G, Hull RD et al. Antithrombotic therapy for venous thromboembolism. Chest 2001; 119: 176S-193S

28. Kakkar VV, Corrigan TP, Fossard DP et al. Prevention of fatal postoperative pulmonary embolism by low-doses of heparin: an international multicenter trial. Lancet 1975; 2: 45-51

29. Koch MO, Smith JA. Low molecular weight heparin and radical prostatectomy: a prospective analysis of safety and side effects prostate cancer. Prostatic Dis 1997; 1: 101-4

30. Lausen I, Jensen R, Jorgensen LN et al. Incidence and prevention of deep venous thrombosis occurring late after general surgery: randomised controlled study of prolonged thromboprophylaxis. Eur J Surg 1998; 164: 657-63

31. Levine MN, Gent M, Hirsh J et al. The thrombogenic effect of anticancer drug therapy in women with stage II breast cancer. $N$ Engl J Med 1988; 318: 404-7

32. Merli G, Spiro TE, Olsson CG et al. Subcutaneous enoxaparin once or twice daily compared with intravenous unfractionated heparin for treatment of venous thromboembolic disease. Ann Intern Med 2001; 134: 191-202

33. Miniati M, Monti S, Pratali L et al. Value of transthoracic echocardiography in the diagnosis of pulmonary embolism: results of a prospective study in unselected patients. Am J Med 2001; 110: 528-35

34. Miniati M, Pistolesi M, Morini C et al. Value of perfusion lung scan in the diagnosis of pulmonary embolism: results of the Prospective Investigative Study of Acute Pulmonary Embolism Diagnosis (PISA-PED). Am J Respir Crit Care Med 1996; 154: $1387-93$

35. Miniati M, Prediletto R, Formichi B et al. Accuracy of clinical assessment in the diagnosis of pulmonary embolism. Am J Respir Crit Care Med 1999; 159: 864-71

36. Morpurgo M, Marzegalli M. Death in pulmonary embolism. In: Morpurgo M. Pulmonary embolism. New York, NY: Marcel Dekker, 1994

37. Moser KM. Venous thromboembolism. Am Rev Respir Dis 1990; 141: 235-49

38. PIOPED Investigators. Value of the ventilation-perfusion scan in acute pulmonary embolism: results of the Prospective Investigation of Pulmonary Embolism Diagnosis (PIOPED). JAMA 1990; 263: 2753-9

39. Raschke RA, Reilly BM, Guidry JR, Fontana JR, Srinivas S. The weight-based heparin dosing nomogram compared with a "standard care" nomogram: a randomized controlled trial. Ann Intern Med 1993; 119: 874-81

40. Ribeiro A, Lindmarker P, Juhlin-Dannfelt A et al. Echocardiography Doppler in pulmonary embolism: right ventricular dysfunction as a predictor of mortality rate. Am Heart J 1997; 134: 479-87

41. Riber C, Alstrup N, Nymann T et al. Postoperative thromboembolism after day-case herniorrhaphy. Br J Surg 1996; 83: 420-1

42. Roncon L, Zonzin P, Giommi L et al. Iter diagnostici e terapeutici nell'embolia polmonare: inchiesta nella Regione Veneto. Cardiologia 1999; 44: 735-41.

43. Samama MM, Cohen AT, Darmon JY, et al. A comparison of enoxaparin with placebo for the prevention of venous thromboembolism in acutely ill medical patients. N Engl J Med 1999; 341: 793-800

44. Sarasin FP, Bounameaux H. Cost-effectiveness of prophylactic anticoagulation prolonged after hospital discharge following general surgery. Arch Intern Med 1996; 131: 694-7

45. Schwartz PJ, Breithardt G, Howard AJ, et al. The legal implications of medical guidelines - a Task Force of the European Society of Cardiology. Eur Heart J 1999; 20: 1152-7

46. Simonneau G, Sors H, Charbonnier B. A comparison of low-molecular-weight heparin with unfractionated heparin for acute pulmonary embolism. N Engl J Med 1997; 337: 663-9

47. Soderdahl DW, Henderson SR, Hansberry KL. A comparison of intermittent pneumatic compression of the calf and whole leg in preventing deep vein thrombosis in urological surgery.J Urol 1997; 157: 1774-6

48. Sors H, Pacouret G, Azarian R, Meyer G, Charbonnier B, Simonneau G. Hemodynamic effects of bolus versus twohour infusion of alteplase in acute massive pulmonary embolism. A randomized controlled multicenter trial. Chest 1994; 106: 712-7

49. Task Force sull'Embolia Polmonare, Società Europea di Cardiologia. Linee guida per la diagnosi e il trattamento dell'embolia polmonare acuta. Ital Heart J Suppl 2001; 2: 161-99

50. Tebbe U, Graf A, Kamke W. Hemodynamic effects of double bolus reteplase versus alteplase infusion in massive pulmonary embolism. Am Heart J 1999; 138: 39-44

51. Todd CJ, Freeman CJ, Camilleri-Ferrante C et al. Differences in mortality after fracture of hip: the East Anglian audit. BMJ 1995; 310: 904-8

52. Turpie AG, Gent M, Cote R et al. A low molecular weight heparinoid compared with unfractionated heparin in the prevention of deep vein thrombosis in patients with acute ischemic stroke: a randomised, double blind study. Ann Intern Med 1992; 117: 353-7

53. Turpie AG, Levine MN, Hirsh J et al. A randomized controlled trial of a low-molecular weight heparin (enoxaparin) to prevent deep-vein thrombosis in patients undergoing elective hip surgery. $N$ Engl J Med 1986; 315: 925-9

54. Vandermeulen EP, Van Aken H, Vermylen J. Anticoagulants and spinal-epidural anesthesia. Anesth Analg 1994; 79: 1165-77 
55. Wray R, Maurer B, Shillingford J. Prophylactic anticoagulant therapy in the prevention of calf vein thrombosis after myocardial infarction. N Engl J Med 1973; 288: 815-7

56. Wysowski DK, Talarico L, Bacsanyi J, Botstein P. Spinal and epidural hematoma and low molecular weight heparin. $N$ Engl J Med 1998; 338: 1774-5

57. Zonzin P, Roncon L, Rinuncini M. Embolia polmonare massiva e controindicazioni alla trombolisi: ruolo della trombectomia meccanica. Ital Heart J Suppl 2000; 1: 1502-3

\section{Parte legale}

1. Cassazione penale, sez. IV, 7 dicembre 2005, sentenza n. 44656

2. Cass. penale, 11 marzo 2004, sentenza n. 11518

3. Corte di Appello di Bari sentenza del 7 aprile 2001

4. Cassazione Penale, 11 marzo 2004, sentenza n. 11518

\section{CORRESPONDING AUTHOR}

Avv.Ennio Grassini, e-mail: info@dirittosanitario.net 